\title{
Research Square \\ Effective Measures Coping with COVID-19 Pandemic Based on fsQCA: Evidence from China
}

\section{Xiaoman Bi}

China University of Geosciences Beijing

Haiyan Liu ( $\sim$ liuhy@cugb.edu.cn )

China University of Geosciences Beijing https://orcid.org/0000-0001-6055-1371

\section{Wei Yang}

China University of Geosciences Beijing

\section{Research Article}

Keywords: COVID-19, emergency measures, effectiveness assessment, fsQCA, emergent infectious diseases

Posted Date: August 6th, 2020

DOI: https://doi.org/10.21203/rs.3.rs-51776/v1

License: (c) (i) This work is licensed under a Creative Commons Attribution 4.0 International License. Read Full License 
Effective measures coping with COVID-19 pandemic based on fsQCA: Evidence from China Xiaoman Bi, Haiyan Liu*, Wei Yang School of Economics and Management, China University of Geosciences,Beijing 100083,China

*Correspondence:liuhy@cugb.edu.cn

\section{Abstract}

Background: Faced with the global outbreak and spread of COVID-19 pandemic, the government of each country has adopted different emergency measures, but the effectiveness assessment of these emergency measures is still lacked. This paper aimed to study the effectiveness of emergency measures for emergent infectious diseases to look for key measures and effective combinations.

1 Methods: We took COVID 19 pandemic management and control measures in China as an example, and selected five high frequency emergency measures including controlling population mobility, patient admission, appropriation of pandemic prevention funds, legal regulations and resumption of production from the perspective of Epidemiology. Using fuzzy set qualitative comparative analysis (fsQCA), we conducted necessary condition analysis and configuration analysis on these measures using official statistical data. And robustness test was performed by changing consistency threshold.

Results: We found that: (1) The consistency of the five emergency measures was less than 0.9 , indicating that none of these measures can be used separately as a necessary condition for the strong pandemic prevention and control effects. (2) There were three combinations of emergency measures to achieve strong pandemic prevention and control effects, with solution consistency of 0.92 and solution coverage of 0.6: when all patients are admitted to hospitals, the 
23 government provides sufficient pandemic prevention funds, and severely crack down on

24 criminal cases; when resume production under the measures of strictly controlling population 25 mobility and severely cracking down on criminal cases; when strictly control population 26 mobility and all patients are admitted to hospitals without resumption production.

27 Conclusions: These findings suggest that fsQCA model can be applied to comprehensive 28 effectiveness assessment of infectious diseases emergency measures. The results of our study 29 not only help various countries prevent and control COVID-19 pandemic and its resurgence, 30 but also have important reference significance for the emergency management of other 31 emergent infectious diseases in the world.

32 Keywords: COVID-19; emergency measures; effectiveness assessment; fsQCA; emergent 33 infectious diseases

\section{1. Background}

In the process of human development, infectious diseases have always been one of human disasters, especially the spread of emergent infectious diseases. The outbreak and spread of COVID-19 has spread to more than 200 countries and regions around the world, with a total of more than 8 million diagnosed cases and nearly 300,000 deaths, which makes it the most serious global crisis since World War II [1]. Scholars have done a lot of research on the spread mechanism and control measures of emergent infectious diseases. The researches related to this

41 article can be summarized into two aspects. 
44 China's emergency management system and prevention and control measures. For example,

45 Wangs et al. [2] found that a sound system of emergency reserve supplies is crucial to the management of public health emergencies by reflecting on the outbreak of SARS and COVID-19 of China, and improving the system of emergency reserve medical supplies should be emphasized in the process of establishing and improving public health emergency response systems. Xu et al. [3] and Zhao et al. [4] analyzed the prevention and control measures taken during the COVID-19 pandemic in Shenzhen and Zhengzhou provinces of China, such as input population control, urban internal population flow control, traffic control, etc., and evaluated the strength and effectiveness of these measures. Others have analyzed the health emergency management system and prevention and control measures of other countries, summed up their successful experience and pointed out the defects. For example, Li [5] comparatively analyzed the overall emergency management system and health emergency management system of typical countries such as the United States and Japan, and make recommendations for China's health emergency management system; Kamenidou et al. [6] took Greek citizens as the research object and found that the most common methods for citizens to prevent COVID-19 are avoiding non-mandatory transportation, contacting with individuals with respiratory symptoms, and individuals of high risk for severe illness; Reis et al. [7] used Brazil, Italy, and South Korea as the typical countries in the initial, peak, and controlled phases of COVID-19, respectively, and analyzed the importance of mitigation policies such as social distancing and isolation policies for controlling the spread of COVID-19. Other countries such as France and Germany have also adopted strict isolation and containment measures such as the "foot ban" and "social ban", and achieved prevention and control results such as a decline in hospital admissions and a 

slowdown in the pandemic. But due to the late start of these policies, the number of infected people in both countries is greater, and the situation of pandemic prevention and control is more severe. Besides, the attitude adopted for the prevention and control of COVID-19 pandemic in the United Kingdom and the United States was mainly the natural law of group immunity and survival of the fittest, resulting in a wide range of infections and deaths.

The other aspect of researches related to this article is the study of methods and models of emergency management for infectious diseases. The predictive model of infectious diseases can provide data support for emergency management policies and reduce socioeconomic harm. The emergency management simulation model can simulate different scenarios and be applied to policy analysis and evaluation. Geng et al. [8] built a SEIR model and added new parameters of latent infection rate and change rate of infected, found that the isolation measures based on strict travel restrictions could slow down the development trend of COVID-19 incidence curve, Similarly, Chinazzi et al. [9] using a global metapopulation disease transmission model, revealed that the travel quarantine of Wuhan delayed the overall COVID-19 epidemic progression in Mainland China and had a more marked effect at the international scale. Additionally, Zhou et al.[10] develop a seeding time and doubling time model to assess risk and response during COVID-19 epidemics, which can help decision makers take decisive action timely.

Compared with other countries in the world, China's early prevention and control of COVID-19 pandemic has been effective. Lai et al. [11] believe that China's combined non-pharmaceutical interventions, including inter-city travel restrictions, early identification and isolation of cases, as well as contact restrictions and social distancing measures, have 
greatly curbed the spread of COVID-19 pandemic in China. However, there are two shortcomings in the existing researches on the emergency management of public health emergencies including emergent infectious diseases. From the perspective of visual angle, most researches focus on the construction and specification of concepts, systems, mechanisms at the macro level, and there are little researches on the effects of specific comprehensive factors management and control measures for emergent infectious diseases. From the perspective of research methods, there are many studies on putting prevention and control measures into the model for analysis, and few studies on the comprehensive evaluation on the effects of management and control measures.

Currently, one of the most popular and effective method for the effectiveness assessment of various management measures is QCA (Qualitative Comparative Analysis). QCA is an emerging research method in the field of social sciences, which was first proposed by sociologist Charles Ragin in the 1980 [12]. Complex causality contained in social phenomena is explored based on Boolean algebra and set theory by case-oriented analysis. With the continuous development of QCA, three analysis techniques include crisp-set QCA (csQCA), fuzzy-set QCA (fsQCA), and multi-value QCA (mvQCA) have gradually formed [12]. Due to QCA has obvious advantages in solving the logical relationship between multiple causal conditions and a specific result, more and more scholars have begun to pay attention to this method. Its applications have penetrated into a host of fields, including innovation management, international business, human resource management, stakeholder management, organizational design, strategic management, knowledge management, entrepreneurial management, and consumer behavior [13]. 
112 for emergent infectious diseases to look for key measures and their effective combinations using fuzzy-set qualitative comparative analysis (fsQCA). Accordingly, from the perspective of

114 Epidemiology, five high-frequency emergency measures such as controlling population mobility, patient admission, appropriation of epidemic prevention funds, legal regulations and resumption of production are selected. In the fsQCA model, the five pandemic emergency measures and emergency management effect were used as condition variables and outcome variables, respectively. Sample data from 31 provinces (autonomous regions and municipalities)

119 in China are input to train an fsQCA optimization model. Through the necessary condition 120 analysis and condition configuration analysis of the model, we find out key measures and their 121 combinations that resulting strong pandemic prevention and control effects and put forward 122 several corresponding policy recommendations. The results of our study not only help various

123 countries prevent and control COVID-19 pandemic and its resurgence, but also have important 124 reference significance for the emergency management of other emergent infectious diseases in 125 the world.

\section{Methods}

\subsection{FsQCA}

FsQCA has quantitative expressions in qualitative analysis, it is good at dealing with the multiple conjunctural outcome evaluation and the cause analysis for the strength or weakness of management effects. Therefore, we use fsQCA to study the effect of COVID-19 pandemic 
emergency measures. The basic thought of fsQCA is to use set theory and Boolean operations as the cornerstones of its methodology, to explore the general characteristics of multiple cases by discussing the membership relationship between sets, and to analyze the set relationship between condition sets and outcome sets of cases $[14,16]$.

The application steps of fsQCA are: (1) Identify the research object, meanwhile determine the outcome variables and screen the appropriate cases. (2) Determine the condition variables, collect and calibrate the data in the unit of a single case sample, analyze the necessary conditions of the single factor, and construct the truth table. (3) Solve the truth table in fsQCA software, check the necessity of a single factor, and then conduct the configurations analysis.

Use consistency to determine whether the logical condition combinations constitute sufficient or necessary conditions for the outcome variable. Use coverage to analyze the ability of logical condition combination to explain the outcome variable [15]. Consistency refers to the degree of consistency of cases with common conditional configuration that belong to a same outcome, and coverage refers to the extent of the set relationships that passes the consistency test interprete the outcome [12].

The model solution software is fs/QCA 3.0 [16].

\subsection{Data and preprocess}

\subsubsection{Variable design and case selection}

The key step in performing fsQCA analysis is the selection of variables. We select the newly confirmed cases from the pandemic notification data released by National Health Commission of the People's Republic of China as the measurement indicators of the pandemic 
prevention and control effect [17]. The selection perspectives of the condition variable are controlling the source of infection, cutting off the route of transmission, protecting susceptible people and resumption of production. The selected time period is the outbreak period of COVID-19 pandemic, which is between January 26 and February 19, 2020.

(1) Controlling the source of infection

In terms of controlling the source of infection, the taken measures are firstly controlling population mobility and secondly patient admission. Population mobility increases the risk of infection, so the sum of newly diagnosed and suspected populations is used as a measure to control population mobility. The measurement index of the patient admission is the number of local designated hospitals and hot clinics for patients with COVID-19.

(2) Cutting off the route of transmission

The transmission routes of COVID-19 are contact transmission and droplet transmission, which mainly depend on person-to-person transmission. Therefore, measures to cut off the transmission route are mainly to prevent crowds from gathering. In the case of controlling population movement, the problem of crowding is prevented naturally.

(3)Protecting susceptible people

The most effective protection measure for susceptible people is vaccination, but for emergent infectious diseases, there is no effective vaccine in the world and it cannot be developed in a short time. Therefore, the protection measures selected for the susceptible people in this paper include two aspects. Firstly, appropriate pandemic prevention funds to conduct epidemic prevention education, health education, disinfection in public places, and improvement of sanitary conditions. Secondly, carry out legal regulations to reduce the 
manufacture and sale of fake medical supplies and crack down behaviors that impede the order

175 of pandemic prevention and control. The measurement of appropriation of pandemic prevention

176 funds uses the number of COVID-19 pandemic prevention funds in various regions. The legal

177 regulation measures are the number of crimes related to the pandemic that have been cracked 178 down during the pandemic.

(4) Resumption of production

Resuming production can maintain the normal operation of the national economy and prevention and control measures are improper, the control of the pandemic may be broke down, so the resumption measures are also very crucial for pandemic control. The measurement index

184 for resumption of production is the change in the number of newly diagnosed people after resumption of work in various regions.

In summary, the condition variables selected in this paper are subdivided into controlling

187 population mobility, patient admission, appropriation of pandemic prevention funds, legal

188 regulations and resumption of production. 31 provinces (autonomous regions and municipalities)

189 in China are used as sample cases to collect and process data.

\subsubsection{Data preprocess}

Data preprocessing in this paper includes two aspects.

Due to the different population distribution and the risk of pandemic, the level of each indicator varies greatly. Therefore, in order to ensure the comparability and reliability of the 
method. Here is the formula:

$$
x_{i}^{\prime}=\frac{x_{i}-|\min |}{|\max |-|\min |}=\frac{x_{i}-|\min |}{R} \in[-1,1]
$$

197

\section{(2)Calibration}

Cases need to be calibrated before qualitative comparative analysis to make the cases interpretable. According to the fuzzy-set (fsQCA) analysis technology, we use the direct calibration method to calibrate the variable data. This method uses three important qualitative thresholds for structured calibration: full membership, full non-membership and the crossover point [18]. We set three qualitative thresholds for the target set based on the lower quartile, upper quartile, and median of the sample data [19]. The measurement index description of each variable and the determination of thresholds are shown in Table 1.

Table 1 Index description and calibration of outcome variable and condition variables

\begin{tabular}{|c|c|c|c|c|}
\hline Variable & Index description & $\begin{array}{l}\text { Fully } \\
\text { In }\end{array}$ & Crossover & $\begin{array}{l}\text { Fully } \\
\text { Out }\end{array}$ \\
\hline $\begin{array}{l}\text { prevention and control } \\
\text { effect }\end{array}$ & Number of newly diagnosed & 0.024 & 0.011 & 0.004 \\
\hline $\begin{array}{l}\text { controlling population } \\
\text { mobility }\end{array}$ & $\begin{array}{l}\text { sum of newly diagnosed and suspected } \\
\text { populations }\end{array}$ & 0.112 & 0.045 & 0.000 \\
\hline patient admission & $\begin{array}{l}\text { number of designated hospitals and hot } \\
\text { clinics for patients with COVID-19 }\end{array}$ & 0.103 & 0.062 & 0.024 \\
\hline $\begin{array}{l}\text { appropriation of } \\
\text { pandemic prevention } \\
\text { funds }\end{array}$ & $\begin{array}{l}\text { number of COVID-19 pandemic } \\
\text { prevention funds }\end{array}$ & 0.289 & 0.157 & 0.068 \\
\hline legal regulations & $\begin{array}{l}\text { number of crimes related to the } \\
\text { pandemic that have been cracked down } \\
\text { during the pandemic }\end{array}$ & 0.112 & 0.024 & 0.012 \\
\hline $\begin{array}{l}\text { resumption of } \\
\text { production }\end{array}$ & $\begin{array}{l}\text { change in the number of newly } \\
\text { diagnosed people after resumption of } \\
\text { work }\end{array}$ & 0.008 & 0.003 & 0.000 \\
\hline
\end{tabular}




\subsection{Necessary condition analysis}

Necessary condition analysis is carried out first before configuration analysis to detect

whether a single variable is a necessary condition for the outcome variable, using consistency

210 and coverage to determine. Consistency refers to the degree of consistency of cases with

211 common conditional configuration that belong to a same outcome, and coverage refers to the

212 extent of the set relationships that passes the consistency test interpret the outcome [12]. Their

213 calculation formulas are as follows, Where $\mathrm{X}$ represents the set of condition variables and $\mathrm{Y}$

214 represents the set of outcome variables. It is generally believed that the consistency detection standard is reached when the consistency score is greater than 0.9 , that is, the variable

constitutes a necessary condition for the outcome [20].

$$
\begin{gathered}
\operatorname{consistency}\left(X_{i} \leq Y_{i}\right)=\sum\left[\min \left(X_{i}, Y_{i}\right)\right] / \sum\left(X_{i}\right) \\
\operatorname{coverage}\left(X_{i} \leq Y_{i}\right)=\sum\left[\min \left(X_{i}, Y_{i}\right)\right] / \sum\left(Y_{i}\right)
\end{gathered}
$$

217 Perform necessary condition analysis on the calibrated data, the results are shown in Table 2.

Table 2 Necessary condition analysis for the strong prevention and control effect of

\begin{tabular}{lll}
\hline Condition Variable & consistency & coverage \\
\hline controlling population mobility & 0.525182 & 0.530743 \\
$\sim$ controlling population mobility & 0.584845 & 0.561953 \\
patient admission & 0.714454 & 0.746732 \\
$\sim$ patient admission & 0.373305 & 0.347752 \\
appropriation of pandemic prevention funds & 0.689567 & 0.651991 \\
$\sim$ appropriation of pandemic prevention funds & 0.392298 & 0.40334 \\
legal regulations & 0.705875 & 0.752181 \\
$\sim$ legal regulations & 0.417251 & 0.382161 \\
resumption of production & 0.700701 & 0.669231 \\
\hline
\end{tabular}




\begin{tabular}{lcc}
\hline$\sim$ resumption of production & 0.390988 & 0.397655 \\
\hline
\end{tabular}

Notes: Wavy lines “ ” indicate"NO/Not”.

According to the results of necessary condition analysis for the strong prevention and

227 is weak. This result shows the complexity of pandemic prevention and control, that is,

228 controlling population mobility, patient admission, appropriation of pandemic prevention funds,

229 legal regulations and resumption of production need to be coordinated with each other to 230 effectively prevent and control COVID-19 pandemic. Accordingly, it is necessary to perform 231 configuration analysis on these condition variables and consider the conjunctural synergistic 232 effect of multiple conditions.

\subsection{Configuration analysis}

Three kinds of solutions can be obtained from conditional configuration analysis of

235 variables: complex solution, intermediate solution and parsimonious solution. Generally, 236 scholars tend to analyze intermediate solutions because they are close to theoretical reality and 237 not too complicated [21]. Consequently, we will focus on the analysis of the intermediate 238 solution.

239 We divide the causal conditions into core conditions and peripheral conditions, and 240 organizes them according to the international form of QCA research results [22], see Table 3. 


\begin{tabular}{|c|c|c|c|c|}
\hline \multirow[b]{2}{*}{ causal condition } & \multicolumn{4}{|c|}{ Configuration } \\
\hline & 1 & 2 & 3 & 4 \\
\hline controlling population mobility & $\otimes$ & O & O & O \\
\hline patient admission & 0 & $\otimes$ & $\mathbf{0}$ & - \\
\hline appropriation of pandemic prevention funds & 0 & - & $\otimes$ & $\bullet$ \\
\hline legal regulations & 0 & 0 & $\otimes$ & 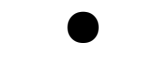 \\
\hline resumption of production & - & 0 & $\otimes$ & $\mathbf{0}$ \\
\hline consistency & 0.921225 & 0.903602 & 0.868545 & 0.933902 \\
\hline raw coverage & 0.275722 & 0.116642 & 0.12116 & 0.286856 \\
\hline unique coverage & 0.20892 & 0.0079245 & 0.0864497 & 0.153252 \\
\hline solution consistency & \multicolumn{4}{|c|}{0.917091} \\
\hline solution coverage & \multicolumn{4}{|c|}{0.601284} \\
\hline
\end{tabular}

The core condition refers to a condition that has an essential meaning to the interpretation of the outcome variable, and appears in both the parsimonious solution and the intermediate solution; the peripheral condition refers to a condition that has no essential influence on the interpretation of the outcome variable and can be replaced, only appears in the intermediate solution. In table3, black circles ( "O” ) indicate the presence of a condition, and circles with a cross-out (“®”) indicate its absence. Furthermore, large circles indicate core conditions, and small circles refer to peripheral conditions. Short horizontal line(“-”)in a solution indicate a "don’t care” situation in which the causal condition may be either present or absent.

Table 3 Configurations of achieving strong pandemic prevention and control effects Notes:Black circles indicate the presence of a condition, and circles with " $\times$ " indicate its absence.

251 Large circles indicate core conditions; small ones, peripheral conditions. Short horizontal lines indicate “don’t care.”

Four paths that can explain the effects of strong pandemic prevention and control are presented in Table 3. The solution consistency is 0.92 , which means that in all the pandemic prevention and control cases that meet these four configurations, $92 \%$ of those are at a high level of epidemic prevention and control effects. The solution coverage is 0.6 , which means that 
257 the four configurations can explain $60 \%$ of the cases of strong pandemic prevention and control

258 effects. The solution consistency and solution coverage both are higher than the critical value,

259 indicating that the empirical analysis is effective.

260

\subsection{Robustness test}

Robustness testing is a crucial analysis step in QCA research [20]. Commonly used robustness testing methods include set theory specific methods and statistical theory specific methods. Zhang and Du [23] believe that QCA research results based on set theory should give priority to set theory specific method to test robustness. Therefore, we choose the method of changing consistency threshold, which is one of the specific robustness test methods of set theory, for the robustness test. We adjusted consistency threshold from 0.8 to 0.85 , and conducted configuration analysis again, and the final result was exactly the same as the configuration analysis result when the consistency threshold was 0.8 , proving that the fsQCA results are robust.

\section{Discussion}

We group configurations which has same core conditions to be the following three situations.

Situation 1, namely configuration 1, the core conditions are patient admission, appropriation of pandemic prevention funds and legal regulations. This situation indicates that a high-intensity pandemic prevention and control effect will be achieved when all patients are admitted to hospitals, the government provides sufficient pandemic prevention funds, and 
severely crack down on criminal cases that violate laws and regulations. The corresponding cases are shown of square in Figure 1. The cases that configuration 1 can explain include three provinces of Zhejiang, Guangdong and Jiangsu. As one of the typical cases, Jiangsu Province has opened 770 designated hospitals and hot clinics for COVID-19 medical treatment, ensuring that diagnosed and suspected patients can be detected and treated effectively in time. Since the pandemic occurred, the financial departments in Jiangsu Province raised funds quickly to ensure the need for pandemic prevention and control can be satisfied. As of March 2, 2020, financial departments at all levels have allocated 5.466 billion yuan, which is mainly used for medical treatment of diagnosed and suspected patients, procurement of equipment and materials, medical personnel subsidy, renovation of isolation wards, medical waste disposal, public area disinfection, and research and development of testing reagents and vaccines, etc. Since late January 2020, the public security office of Jiangsu Province have investigated and dealt with 3399 criminal cases and 11248 administrative cases involving COVID-19 pandemic. Eight major criminal cases in the province have decreased by $38 \%$ year-on-year, and no influential major vicious cases have occurred, which has made outstanding contributions to maintaining the order of pandemic prevention and control. Under the three-pronged approach, the number of COVID-19 newly diagnosed cases in Jiangsu Province from 23 daily on January 27 to 0 on February 19, strongly illustrating the obvious prevention and control effect brought by these measures.

Situation 2, including configuration 2 and configuration 4, the core conditions are controlling population mobility, legal regulation and resumption of production. This shows that under the measures of strictly controlling population mobility and severely cracking down on 
criminal cases that violate laws and regulations, resumption of production can still achieve a high-intensity pandemic prevention and control effect, and it has not brought a negative impact on pandemic prevention and control. Measures to control population movements include mandatory isolation of diagnosed and suspected patients, strict prohibition of residents dropping around, dinners, gatherings, and strict 14-day home isolation for returnees. Taghrir et al. [24] also proved the key role of large-scale isolation in the prevention and control of COVID-19 pandemic. Compared with configuration 2, configuration 4 also emphasizes the peripheral significance of the measure for appropriation of pandemic prevention funds. The corresponding case of configuration 2 is Anhui (as shown of circle in Figure 1), and the corresponding cases of configuration 4 include Anhui, Henan, Shandong and Sichuan (as shown of triangle in Figure 1). Taking Henan as a typical case, the number of newly diagnosed and suspected in Henan has decreased by 15 and 906 respectively in the next three days after government of Henan Province issued the requirement of "strictly implement isolation measures and immediately stop public gathering activities with obvious cross infections" on February 12. Henan Province also strongly cracks down on pandemic-related crimes, focusing on crimes that disturb medical order, violently hurt medical staff, and interfere in pandemic prevention work, as well as manufacture and sale of counterfeit and shoddy medical items, and fraud of pandemic-related supplies, and crimes that raise prices, hoard medical items, and make rumors. As of March 12, Henan public security office punished a total of 6,757 criminal cases involving COVID-19 pandemic. Regarding resumption of production, enterprises in Henan Province have started to resume work successively since February 10, with the prevention and control of the pandemic on the one hand and the resumption of production on the other hand supported by a number of 
321 measures. Instead of causing a rebound in the pandemic, the number of pandemic cases 322 continued to decline, which can be confirmed by the number of confirmed cases fell by 17 three 323 days after resuming production. In addition, Henan Province's finances have cumulatively 324 allocated 3.841 billion yuan for pandemic prevention and control till February 13, which 325 effectively ensures the demand for pandemic prevention and control.

Situation 3, namely configuration 3 , the core conditions are controlling population 327 mobility, patient admission and non-resumption of production. This shows that in the case of 328 strict control of population mobility, all patients are admitted to hospitals and no resumption 329 production, a high-intensity pandemic prevention and control effect can be achieved. However, 330 in order to ensure economic operation and basic people's livelihood needs, all parts of the 331 country have begun to resume production, so configuration 3 has no specific corresponding 332 case to analyze. But it is generally acknowledged that under the measures of controlling the 333 source of infection (controlling population movement and patient admission) and avoiding the 334 second outbreak of pandemic (not resuming production), a high-intensity pandemic prevention 335 and control effect will inevitably be achieved. 


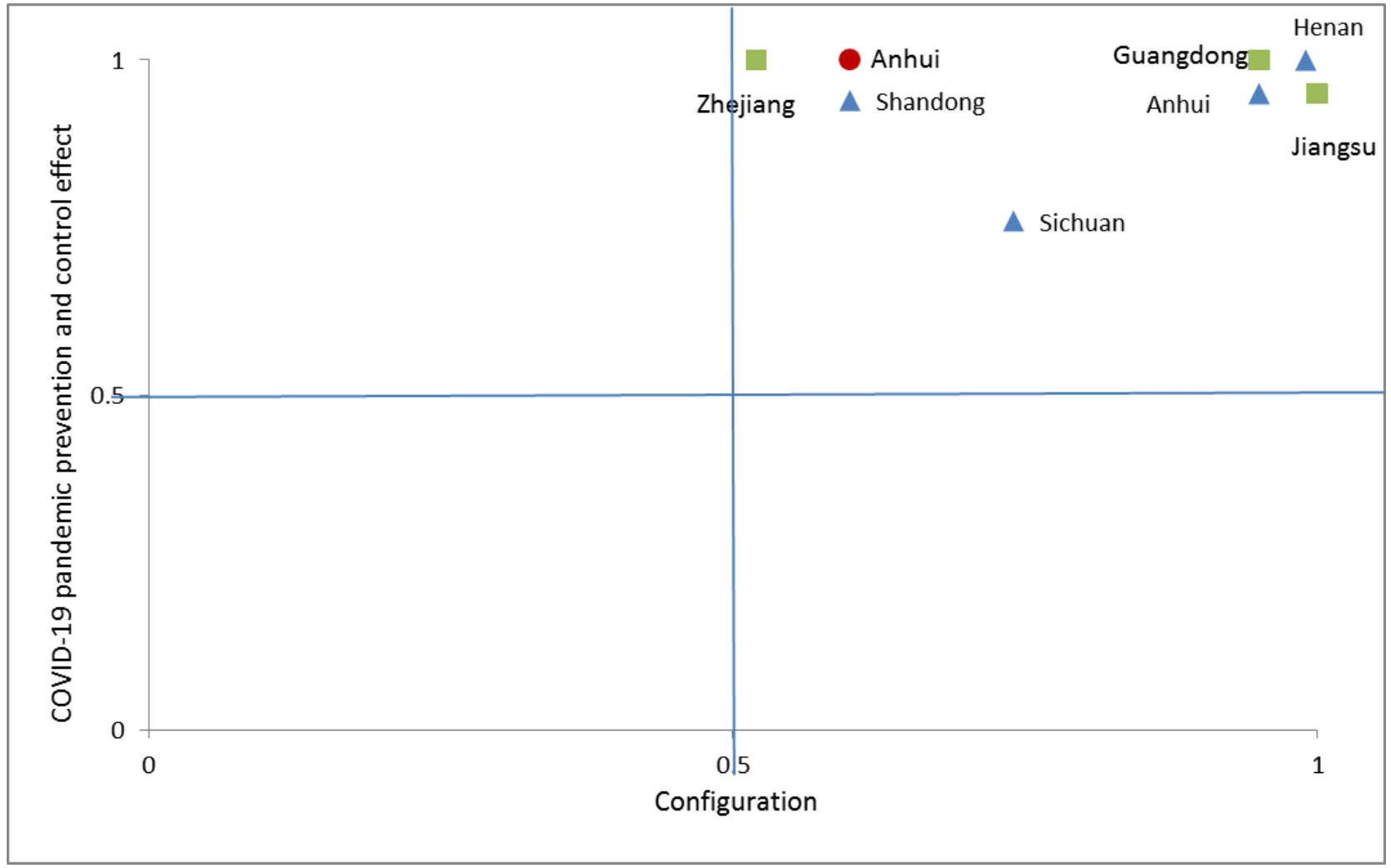

Figure 1 Corresponding cases of configurations configuration 2 , triangles indicate configuration 4 .

Although we have studied the effects of emergency measures for emergent infectious

341 diseases, there are still some limitations of our study. First, we don't cover all pandemic

342 prevention and control measures such as external assistance, vaccination, etc. Future research

343 can take other emergency measures in to consideration from different perspectives and discuss

344 key measures and combinations. Second, the implementation of specific policies will be

345 different more or less due to differences in systems and cultural customs among countries.

346 Therefore, the institutional and cultural differences among countries should be taken into

347 account in future research to extract commonalities and apply to the emergency management of

348 global infectious diseases better. 
We took COVID-19 pandemic in China as an example, from the perspective of 351 Epidemiology, five high-frequency emergency measures including controlling population mobility, patient admission, appropriation of pandemic prevention funds, legal regulations and resumption of production are selected. Using fuzzy set qualitative comparative analysis (fsQCA), we studied the effects of emergency measures for emergent infectious diseases to look for key measures and their effective combinations. Four main conclusions we concluded are as 356 follows. pandemic prevention and control effects, or the ability to independently explain strong pandemic prevention and control effects is weak. In other words, the emergency management of infectious diseases cannot be completed by a certain department or a certain measure, but it needs collaboration of various subjects including governments, professional institutions and social organizations in order to obtain efficacious prevention and control effect.

(2) The government should increase the input of pandemic prevention funds to ensure that pandemic prevention needs such as medical treatment, procurement of medical materials, and vaccine research and development are met. Timely expansion and reconstruction of treatment hospitals should be carried out to ensure that patients are tested and treated immediately. It also 367 should severely crack down on illegal acts that disturb the medical order and interfere with 368 pandemic prevention, such as manufacturing and selling counterfeit and shoddy medical 369 supplies, raising prices, and spreading rumors. 
down on criminal cases that violate laws and regulations, resumption of production can still achieve a high-intensity pandemic prevention and control effect, and it will not bring a negative impact on pandemic prevention and control. Measures to control population movement include mandatory isolation of diagnosed, suspected patients and close contacts, banned gathering activities such as dinners, parties, and exhibitions, and temporarily closing places other than the public places necessary to guarantee the lives of the masses. You can also formulate relevant laws or issue legally effective notices to ensure the smooth implementation of the above measures. In addition, with proper protection measures, a reasonable and orderly resumption of production will not have a negative impact on the prevention and control of infectious diseases, instead it can increase the production to alleviate the shortage of medical supplies, protect the residents normal life and maintain a stable social order.

(4) In the case of strict control of population mobility, all patients are admitted to hospitals and no resumption production, a high-intensity pandemic prevention and control effect can be achieved. However, due to the inability to resume production, the supply of medical items, the guarantee of residents' normal life and the stability of social order will be affected. Consequently, it is recommended to adopt a differentiated combination of prevention and control measures unless the pandemic spreads rapidly or is already difficult to control.

These conclusions suggest that fsQCA model can be applied to comprehensive effectiveness assessment of infectious diseases emergency measures. Moreover, efficacious prevention and control effect of infectious diseases emergency management can be achieved only when various subjects including governments, professional institutions and social organizations and various measures carry out jointly. The results of our study not only help 

various countries prevent and control COVID-19 pandemic and its resurgence, but also have 394 important reference significance for the emergency management of other emergent infectious 395 diseases in the world.

\section{6.Declarations}

397 Ethics approval and consent to participate

Not applicable.

\section{Consent for publication}

$400 \quad$ Not applicable.

401 Availability of data and materials

402

The datasets used and analysed during the current study are available from the 403 corresponding author on reasonable request.

\section{Competing interests}

The authors declare that they have no competing interests.

$406 \quad$ Funding

This work was supported by the Special fund of Ministry of education of the People's

408 Republic of China (grant number 26520200 01) and National Natural Science Foundation of 409 China (NSFC) (grant number 7167 3256). The study sponsor had no role in study design; in the 
410 collection, analysis, and interpretation of data; in the writing of the report; and in the decision to 411 submit for publication.

\section{Authors' contributions}

414 data. X.B established the model and wrote the first paper. All authors read and approved the

415 final manuscript. H.L. provided project funding support.

416

\section{Acknowledgements}

Not applicable.

\section{Authors' information}

421 the official policy, or endorsement of their affiliated institutions.

\section{$423 \quad$ Reference}

424 1. Boccaletti S, Ditto W, Mindlin G, Atangana A. Modeling and forecasting of epidemic

425 spreading: The case of Covid-19 and beyond. Chaos, Solitons and Fractals. 2020;135:109794.

2. Wang X, Zhang X, He J. Challenges to the system of reserve medical supplies for public health emergencies: Reflections on the outbreak of the severe acute respiratory syndrome 
3. Xu R, Wang J, Ye S, Wang X. Analysis of prevention measures in Shenzhen based on SIQR model during the novel coronavirus pneumonia. Shenzhen Daxue Xuebao (Ligong Ban)/Journal Shenzhen Univ Sci Eng. 2020;37(3):257-64.

4. ZHAO H, Jiachen W, Shuang W, Yaxin L, Guanghui L, Changhong M. The Risk Assessment of Covid-2019 Epidemic in Metropolis and Precise Prevention and Control Measures: A Case Study of Zhengzhou City. Econ Geogr. 2020;40(4):103-9,124.

5. Li X. Study on the countermeasures to improve the national public health emergency management system. Adm Reform. 2020;(4):13-21.

6. Kamenidou IE, Stavrianea A, Liava C. Achieving a Covid-19 Free Country: Citizens Preventive Measures and Communication Pathways. Int J Environ Res Public Health.

7. Reis RF, de Melo Quintela B, de Oliveira Campos J, Gomes JM, Rocha BM, Lobosco M, et al. Characterization of the COVID-19 pandemic and the impact of uncertainties, 2020;17(13):6-10. mitigation strategies, and underreporting of cases in South Korea, Italy, and Brazil. Chaos, Solitons and Fractals . 2020;136:109888.

8. GENG H, XU A, WANG X, ZHANG Y, YIN X, MA M, et al. Analysis of the role of current prevention and control measures in the epidemic of Corona Virus Disease 2019 based on SEIR model. J Jinan Univ Nat Sci \& Med Ed. 2020;41(2):175-80.

9. Chinazzi M, Davis JT, Ajelli M, Gioannini C, Litvinova M, Merler S, et al. The effect of travel restrictions on the spread of the 2019 novel coronavirus (COVID-19) outbreak. Science (80- ). 2020;368(6489):395-400. 
10. Zhou L, Liu JM, Dong XP, McGoogan JM, Wu ZY. COVID-19 seeding time and doubling time model: An early epidemic risk assessment tool. Infect Dis Poverty. 2020;9(1):1-9.

11. Lai S, Ruktanonchai NW, Zhou L, Prosper O, Luo W, Floyd JR, et al. Effect of non-pharmaceutical interventions to contain COVID-19 in China. Nature . 2020; doi: 10.1038/s41586-020-2293-x.

12. Du Y, Jia L. Configuration perspective and qualitative comparative analysis (QCA): a new path for management research. Manage World. 2017;(6):155-67.

13. Zhang C, Zheng X, Wang F. The Application of Qualitative Comparative Analysis (QCA) in Configuration Research in Business Administration Field:Commentary and Future Directions. Foreign Econ Manag. 2017;39(4):68-83.

14. XIA X, HE J, LIU J. Research Logic of Qualitative Comparative Analysis: On Its Implications for Economic Management Research. J Financ Econ. 2014;40(10):97-107.

15. Rihoux B, Ragin CC. Configurational comparative methods:qualitative comparative analysis (QCA) and related techniques. Thousand Oaks: Sage; 2009.

16. Ragin C, Sean D. Fuzzy-Set/Qualitative Comparative Analysis 3.0. Irvine, California: Department of Sociology, University of California; 2016.

17. National Health Commission of the People's Republic of China. http://www.nhc.gov.cn/ Accessed 21 May 2020.

18. Ragin C. Redisigning Social Inquiry: Fuzzy Sets and Beyond .University of Chicago Press; 2008.

19. Coduras A, Clemente JA, Ruiz J. A novel application of fuzzy-set qualitative comparative analysis to GEM data. J Bus Res. 2016;69(4):1265-70. 
473 20. Schneider CQ, Wagemann C. Set-theoretic methods for the social sciences: A guide to qualitative comparative analysis. Cambridge University Press; 2012.

475 21. Crilly D, Zollo M, Hansen MT. Faking it or muddling through understanding decoupling 476 in response to stakeholder pressures. Acad Manag J. 2012;55(6):1429-48.

477 22. Fiss PC. Building better causal theories: A fuzzy set approach to typologies in organization 478 research. Acad Manag J. 2011;54(2):393-420.

479 23. Zhang M, Du Y. Qualitative Comparative Analysis(QCA)in Management and Organization Research:Position,Tactics, and Directions. Chinese J Manag. 2019;16(9):1312-23.

24. Taghrir MH, Akbarialiabad H, Marzaleh MA. Efficacy of mass quarantine as leverage of health system governance during COVID-19 outbreak: A mini policy review. Arch Iran Med. 2020;23(4):265-7. 
Figures

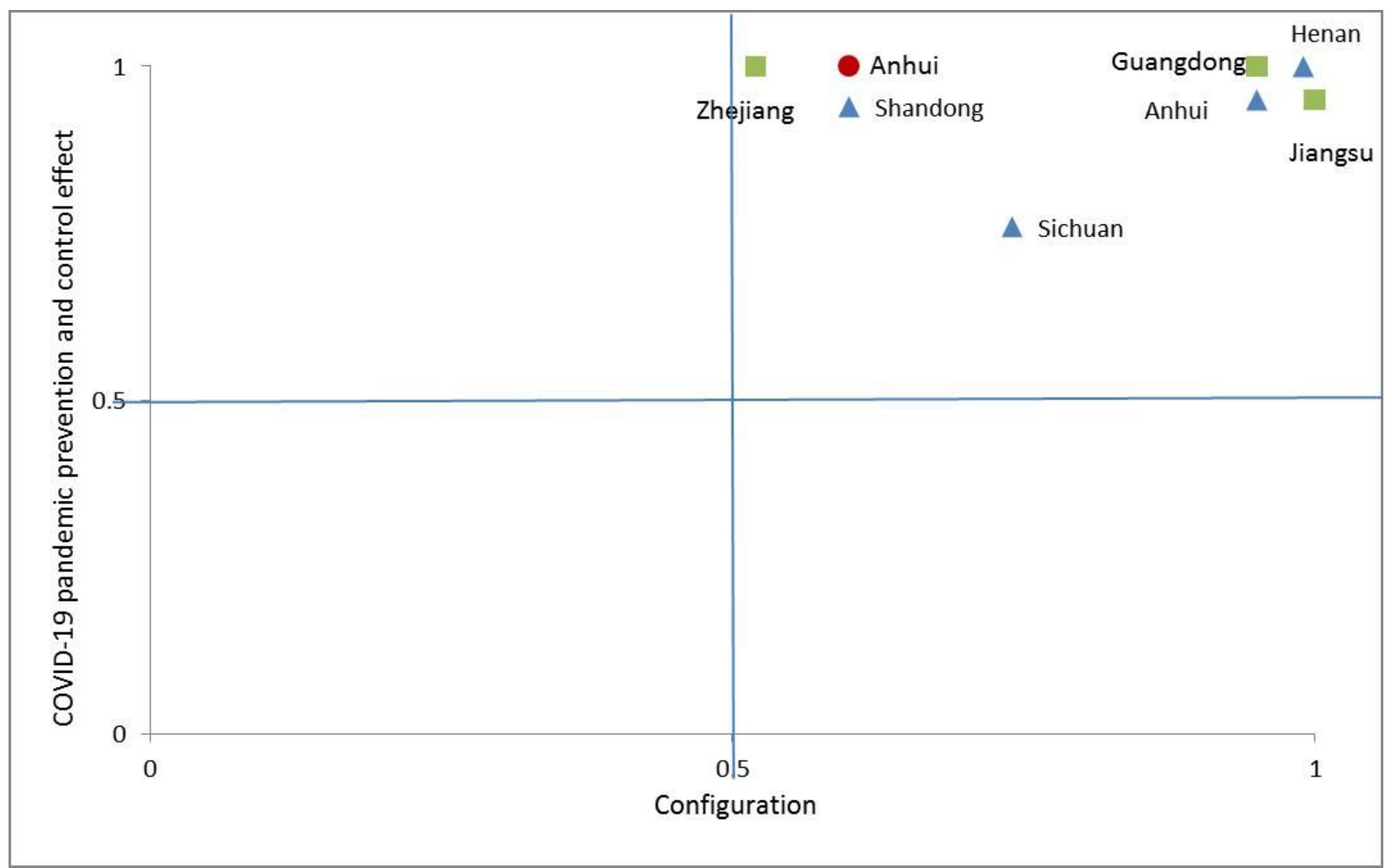

Figure 1

Corresponding cases of configurations 